\title{
Study on Production, Extraction and Analysis of Polyhydroxyalkanoate (PHA) from Bacterial Isolates
}

\author{
Paramjeet Khandpur, Jabeen E.T, K.V.L Rohini, Varaprasad Y, \\ Laxminarayana B \\ Department of Biotechnology, Visvesvaraya Technological University, Karnataka, India.
}

\begin{abstract}
Polyhydroxyalkanoates (PHAs) are polyesters of hydroxyalkanoates synthesized by various bacteria as intracellular carbon and energy storage compounds and accumulated as granules in the cytoplasm of cells. In this work, we have screened the best micro organism among Bacillus subtilis and Pseudomonas aeruginosa and also have screened the best cheap carbon sources among Rice bran, Paddy husk, pigeon pea waste, Sugarcane baggasse, and waste frying oil. The study of production of PHA is done in combination of the best screened substrate as well as the micro organism.
\end{abstract}

Keywords: Polyhydroxyalkanoates, biodegradable polymer, bioplastic.

\section{Introduction}

The accumulation of plastic wastes has become a major concern in terms of the environment(1). Since synthetic plastics marked their debut in the 1950s, they have emerged to be among the most needed material in our daily life(2). The extensive usage of petrochemical plastics due to their versatile properties especially durability is causing severe problem in waste management affecting the aesthetic quality of cities, water bodies and natural areas(3). Problems concerning the global environment have created much attention in developing eco-friendly products .Biopolymers are one product that can help to overcome problems caused by petrochemical polymers. Biopolymers are generated from renewable natural sources and are often biodegradable and nontoxic(4).

Polyhydroxyalkanoates (PHAs) are biodegradable polyesters synthesized by various bacteria They represent products with biotechnological importance due to their special properties. They are accumulated intracellular as carbon and energy reserves under certain conditions(5).

PHA being biodegradable and biocompatible has application that varies from industries to medical therapeutics. Microorganisms accumulate PHA as an intracellular reserved material in response to imbalance in the growth environment where a suitable carbon source is present in excess and one or more nutrients are limiting, example, nitrogen, phosphorus, sodium, oxygen, magnesium, manganese.(6)

PHA production from renewable carbon sources either by microbes or plants has become a valuable commercial prospect due to lower carbon feedstock costs, since PHAs were first identified as environment friendly biodegradable plastics(1)

\subsection{Collection of organism and subculturing}

\section{Materials and Methods}

The organisms Pseudomonas aeruginosa and Bacillus subtilis were collected from Biogenics research centre, Hubli. The subculturing of organisms were done in $25 \mathrm{ml}$ of nutrient broth. The nutrient broth contained $0.25 \mathrm{gm}$ peptone, $0.25 \mathrm{gm} \mathrm{NaCl}$ and $0.125 \mathrm{gm}$ yeast extract in $25 \mathrm{ml}$ distilled water.

The media was sterilized at $120^{\circ} \mathrm{c}$ and $15 \mathrm{lb}$ pressure. The media was cooled to room temperature and inoculated with the bacterial samples under laminar air flow. Two sets of media were prepared for these two samples and were incubated at room temperature on rotary flask shaker at $150 \mathrm{rpm}$ for $72 \mathrm{hours}$. The graph is shown in figure 1.

\subsection{Screening of microorganisms}

\subsubsection{Mineral media preparation}

The mineral media was prepared with the following composition: $0.01 \mathrm{gm}$ yeast extract, $0.1 \mathrm{gm}$ substrate(dextrose), $0.64 \mathrm{gm} \mathrm{NaHPO} .7 \mathrm{H}_{2} \mathrm{O}, 0.15 \mathrm{gm} \mathrm{K}_{2} \mathrm{HPO}_{4}, 0.025 \mathrm{gm} \mathrm{NaCl}, 0.05 \mathrm{gm} \mathrm{NH} 4 \mathrm{Cl}, \mathrm{pH} 7,1 \mathrm{ml}$ of $1 \mathrm{M} \mathrm{MgSO}_{4}$ stock solution, $1 \mathrm{ml}$ of $0.1 \mathrm{M} \mathrm{CaCl}_{2}$ stock solution in $50 \mathrm{ml}$ distilled water(7). Media was sterilized and inoculated with each bacterial sample in separate flasks and were incubated on rotary flask shaker at 150 rpm for 72 hours at room temperature. 


\subsubsection{Microscopic observation}

Microscopic analysis of heat- fixed cells was done after staining with crystal violet for 10sec and destaining with running water for $10 \sec (8)$.The clumps were observed under compound microscope, as shown in figure 2 .

\subsection{3. $\mathrm{N}_{2}$ estimation and extraction}

The samples were centrifuged at $10,000 \mathrm{rpm}$ for 10 minutes. The cell pellets were taken for extraction and the supernatant was taken for $\mathrm{N}_{2}$ estimation.

For $\mathrm{N}_{2}$ estimation Nesslers reagent was used and OD was measured at $500 \mathrm{~nm}$. (9)The graph was plotted by using standard nitrogen values as shown in figure 3 .

For extraction the cell pellets were washed with distilled water and dried at $90^{\circ} \mathrm{C}$ in incubator for $3 \mathrm{hrs}$. Later, cell pellets were collected from tubes by scrapping and weighed. Further, $0.6 \mathrm{ml} \mathrm{of}(9 \mathrm{ml}$ methanol $+0.3 \mathrm{ml} \mathrm{H}_{2} \mathrm{SO}_{4}+1 \mathrm{ml}$ ) mixture was added into the vials containing $0.01 \mathrm{gms}$ of dried cell pellets and heated at $90^{\circ} \mathrm{c}$ for 2 hours. The mixture was then cooled to room temperature. Then $0.6 \mathrm{ml}$ of chloroform and $0.3 \mathrm{ml}$ of distilled water was added and mixed slowly for cell separation. Centrifugation was carried out at $8000 \mathrm{rpm}$ for $1 \mathrm{~min}$, the top chloroform layer containing PHA was collected into a different vial and was taken for analysis using GC-MS shown in figure4.(10)

Gas chromatography mass spectral (GC-MS) was performed with SHIMADZU QP2010S (KUD, Dharwad).The mass spectrometer was auto tuned with decafluorotriphenylphosphine and a70 x $106 \mathrm{eV}$ fragmentation energy. Spectra were recorded at a scan speed of 380atomic mass units (AMU)/s (4 samples per $0.1 \mathrm{AMU}$ ) with a 0.5 -s delay between scans of 50 to $500 \mathrm{AMU}$. The spectrometer was operated in the peak finder mode at an electron multiplier voltage of $1,800 \mathrm{~V}$. The threshold of detectability was 200 linear counts.

\subsection{Screening of substrate}

The easily available cheap carbon sources like rice husk, paddy waste, sugarcane baggasse, pigeon pea waste and waste frying oil were collected from the villages of Karnataka (India). The substrates were grinded into fine powder (except oil). Hydrolyzation of these substrates were done by adding $50 \mathrm{ml}$ of mixture containing $750 \mu \mathrm{l}$ of $\mathrm{H}_{2} \mathrm{SO}_{4}$ in $100 \mathrm{ml}$ distilled water to each $50 \mathrm{gms}$ of powdered substrates and diluted to 1:4 ratio. The flasks containing hydrolyzed carbon were autoclaved and these samples were squeezed using muslin cloth and the filtrate was taken for centrifugation $(10,000 \mathrm{rpm}$ for $10 \mathrm{~min})$. The supernatant was used as carbon source in mineral media for the production of PHA in combination with Pseudomonas aeruginosa. Further procedure for production and extraction of PHA was same as followed in the screening of microorganisms. Analysis was done using GC-MS, the graphs are as shown in figure 5.

\subsection{Effect of carbon source}

Carbon source plays very important role in increasing the concentration of PHA, hence a study of effect of carbon source was performed by increasing the concentration of hydrolyzed carbon source 5 times and 10 times of that used above for the screening of carbon source. GC- MS was performed for both the samples. Graph is shown in figure 6.

\subsection{Growth rate studies of organism:}

\section{RESULTS AND DISCCUSSION}

The growth behavior of individual organism was observed by preparing the nutrient broth, inoculation, incubation and determination of optical density at different time intervals.

The growth pattern exhibited by the individual organism is depicted in the following graph which clearly marks higher growth rate of Pseudomonas aeruginosa over Bacillus subtilis. 


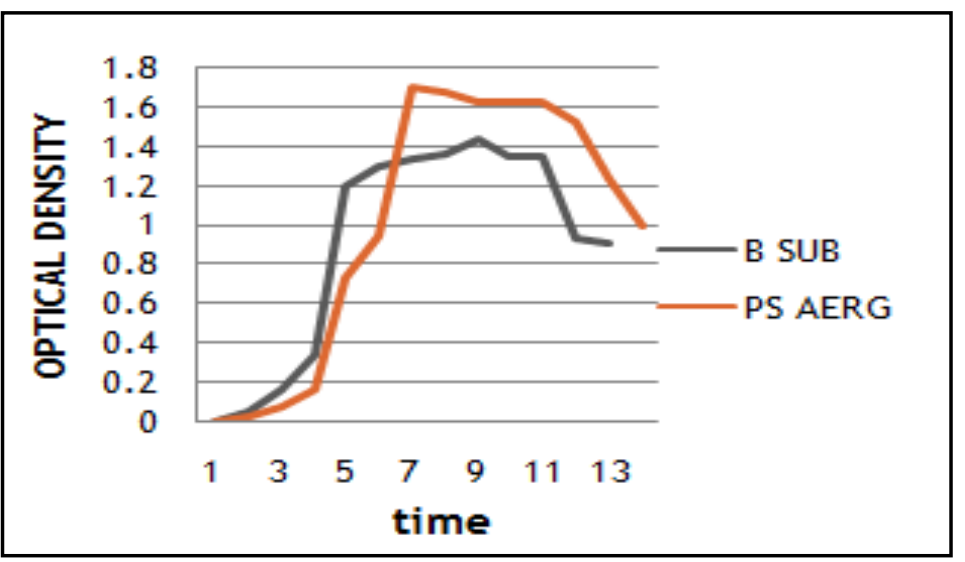

Fig. 1 Growth rate studies of organisms

\subsection{Nitrogen estimation:}

It has been reported that the nitrogen concentration in bacteriological media highly influences the production of intracellular PHAs (Khanna and Srivastava 2005b). From the experimental analysis and comparison of standard nitrogen graph it was found that about $616 \mu \mathrm{g}$ and $328 \mu \mathrm{g}$ of nitrogen was present in the organisms Pseudomonas aeruginosa and Bacillus subtilis.

Table1: Estimation of nitrogen

\begin{tabular}{|l|l|l|l|l|l|}
\hline SI No & Sample(ml) & H2O(ml) & $\begin{array}{l}\text { Neslers reagent } \\
(\mathbf{m l})\end{array}$ & & OD(500nm) \\
\hline 1 & 00 & 2.7 & 0.3 & INCUBATION & 00 \\
\hline 2 & 0.2 & 2.5 & 0.3 & AT ROOM TEMP & 0.317 \\
\hline 3 & 0.2 & 2.5 & 0.3 & FOR 20 MIN & 0.592 \\
\hline
\end{tabular}

Table2: Standard nitrogen table

\begin{tabular}{|l|l|}
\hline Conc. of ammonia $(\boldsymbol{\mu g})$ & OD at $\mathbf{5 0 0} \mathbf{~ n m}$ \\
\hline 00 & 00 \\
\hline 20 & 0.16 \\
\hline 40 & 0.31 \\
\hline 60 & 0.45 \\
\hline 80 & 0.59 \\
\hline 100 & 1.22 \\
\hline
\end{tabular}




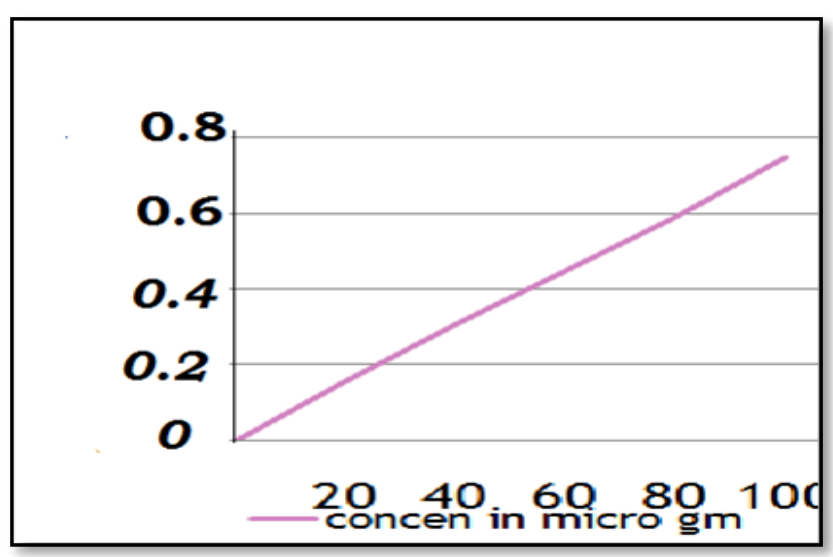

fig: Standard nitrogen curve

\section{3. (a)Microscopic observation:}

Incubation of bacterial samples for 72 hours and staining it with crystal violet revealed the dark clumps of the violet colored cells under microscopic examination , thus qualitatively confirming the production of PHA polymer by the organisms(7).

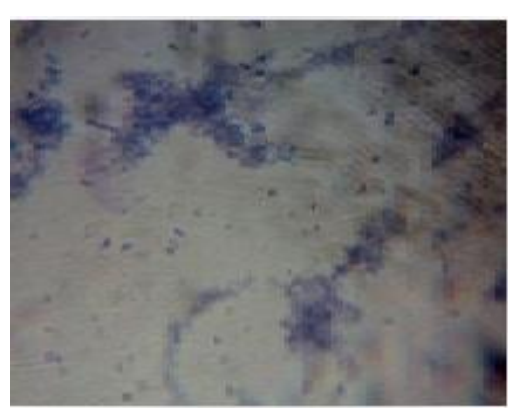

Fig: clumps of Pseudomonas aeruginosa

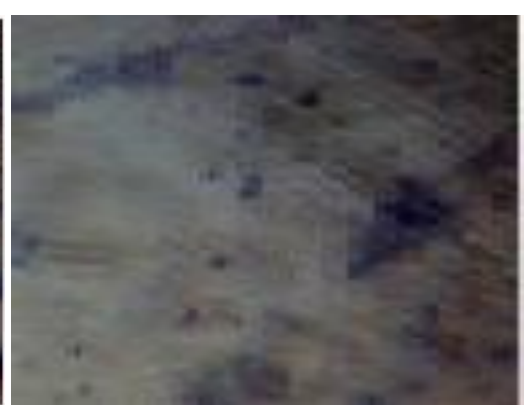

Fig: clumps of Bacillus subtilis

(b)Gas chromatography analysis:

Screening of microorganisms

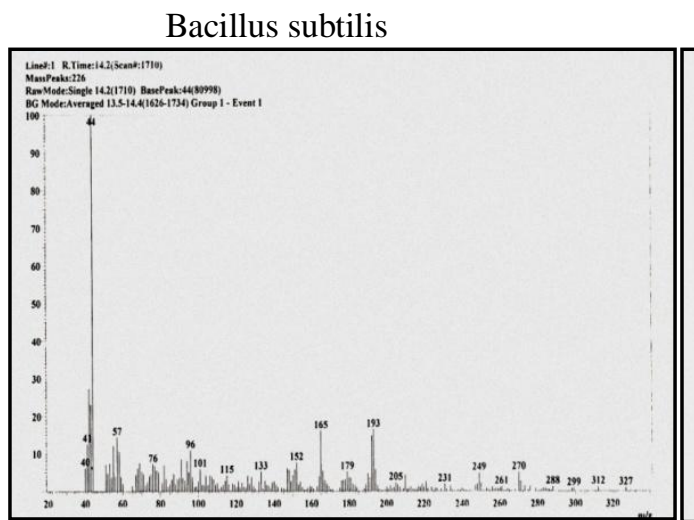

Pseudomonas aeruginosa

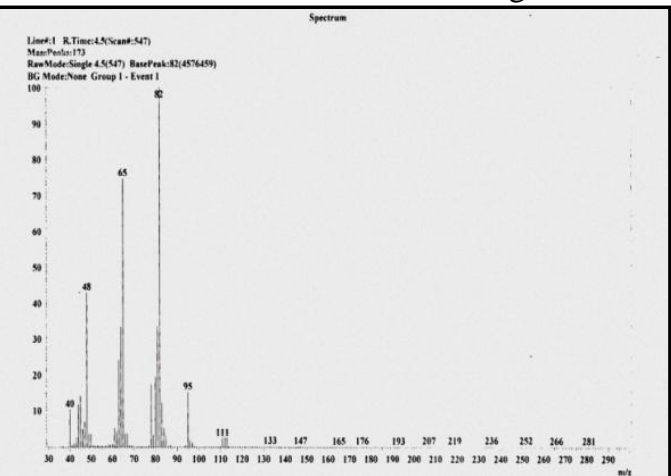

Screening of substrates 


\section{Screening of substrates}

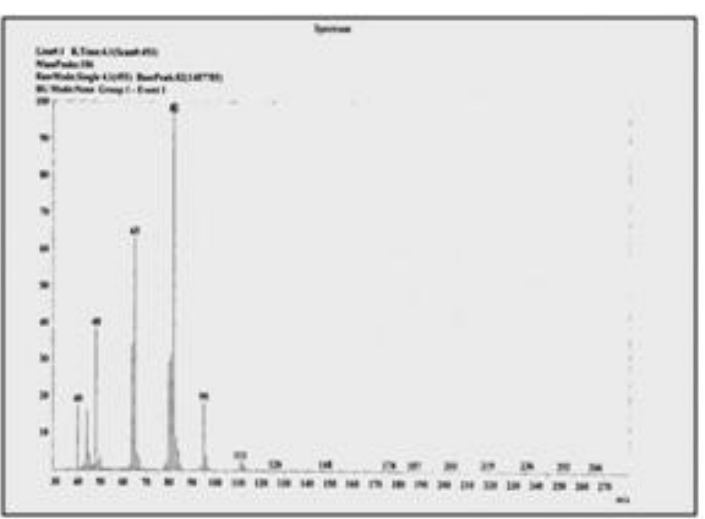

Paddy waste

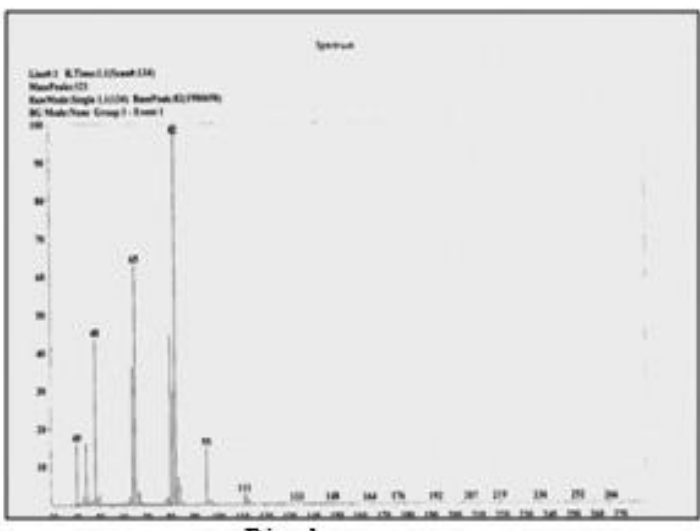

Rice bran

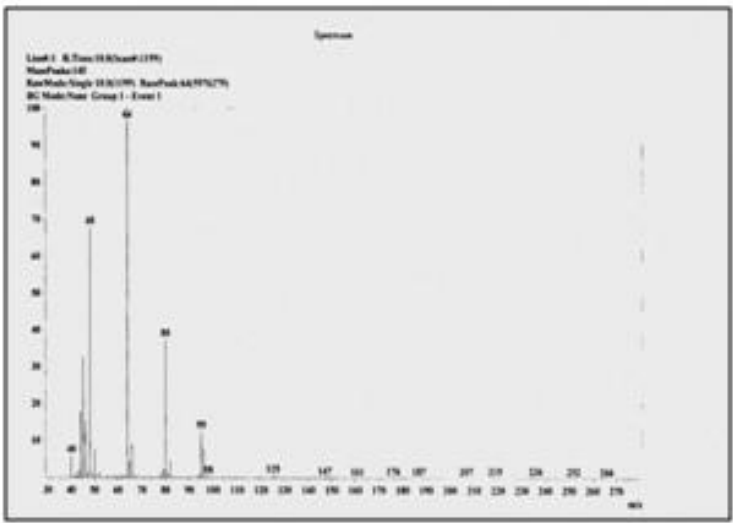

pigeon pea waste

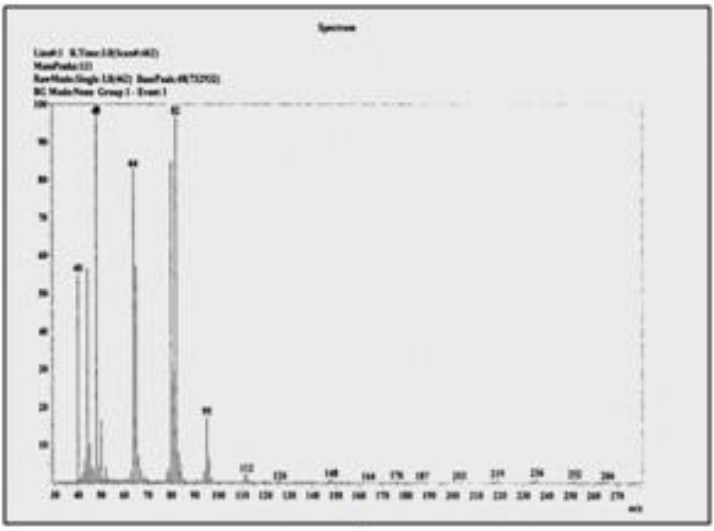

sugarcase bagasse

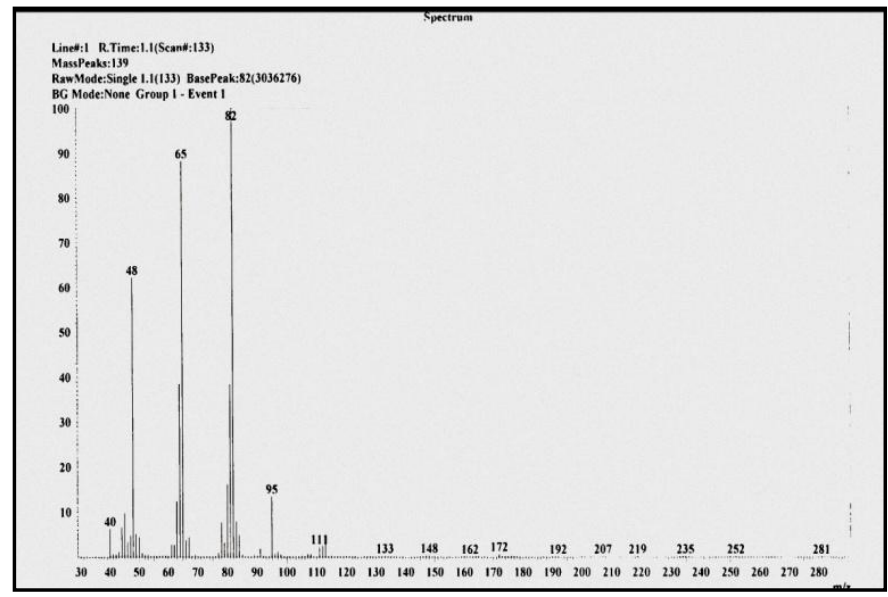

Waste frying oil 


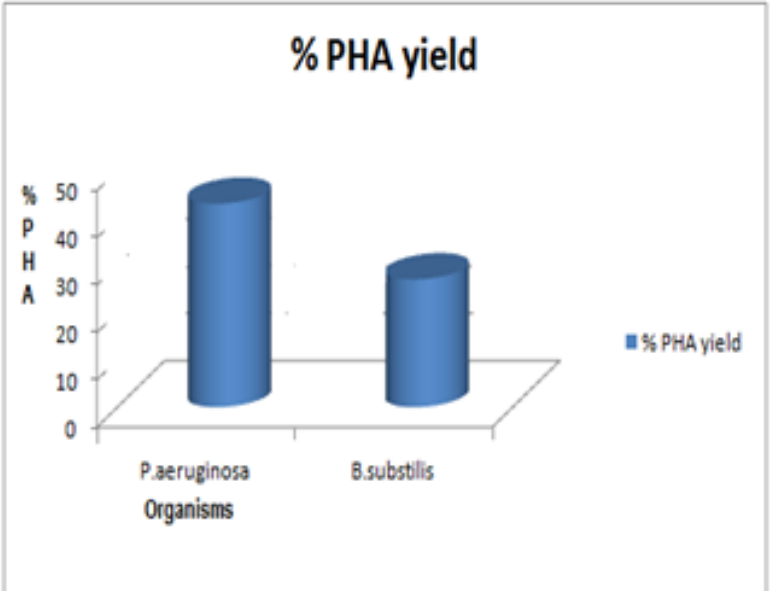

Percentage PHA yield by P.aeruginosa and B.subtilis

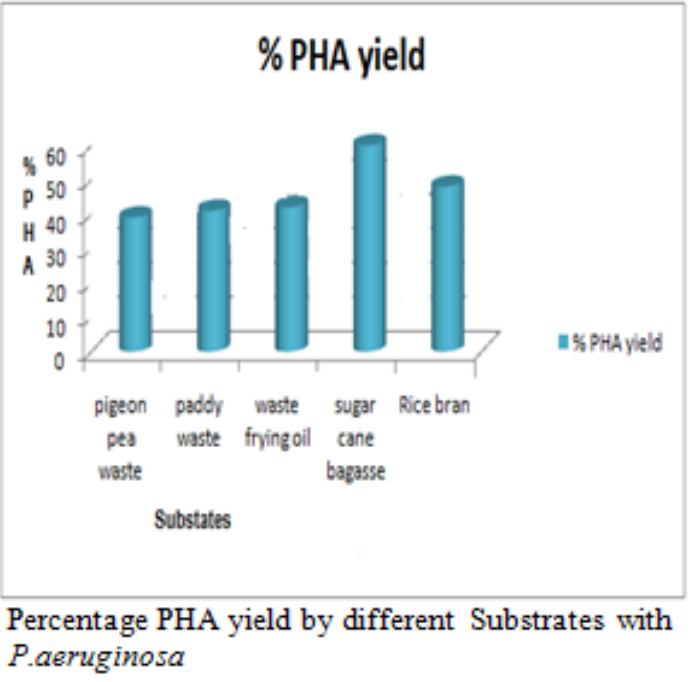

From the GC-MS graphs the height of the peaks indicates the concentration of PHA present in the different organisms. According to the literature survey the molecular weight of PHA should not exceed 120 $\mathrm{m} / \mathrm{z}$. The graph of Bacillus subtilis showed many different peaks and the peaks exceeded $120 \mathrm{~m} / \mathrm{z}$. Hence it indicates the presence of other lipid contaminants. Where as in the graph of Pseudomonas aeruginosa the peaks are clear and are not exceeding $120 \mathrm{~m} / \mathrm{z}$. The result in the form of the bar graph clearly shows the presence of the percentage of PHA in both the organisms.

The percentage of PHA in Pseudomonas aeruginosa was found to be $50 \%$ and the same in Bacillus subtilis is $30 \%$. Hence we conclude that among the two, Pseudomonas aeruginosa can produce more PHA and was screened as the best organism for the production of polyhydroxyalkanoate.

From the graph Pseudomonas aeruginosa with the combination of five different substrates i.e pigeon pea waste, paddy waste, waste frying oil, sugarcane baggasse and rice bran produced about 39\%, $41 \%, 42 \%, 60 \%$ and $48 \%$ of PHA.

There by we conclude that Pseudomonas aeruginosa in combination with Sugarcane baggasse produce the maximum concentration of PHA. Hence sugarcane baggasse was screened as the best substrate for the production of PHA with Pseudomonas aeruginosa.
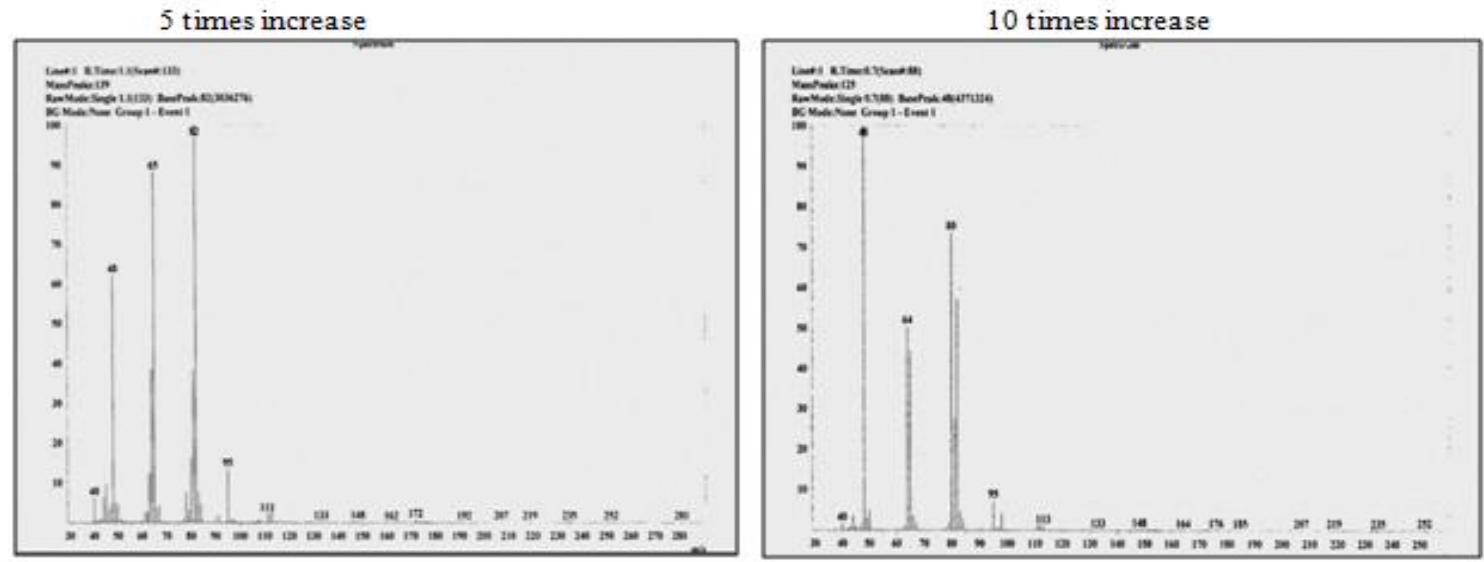


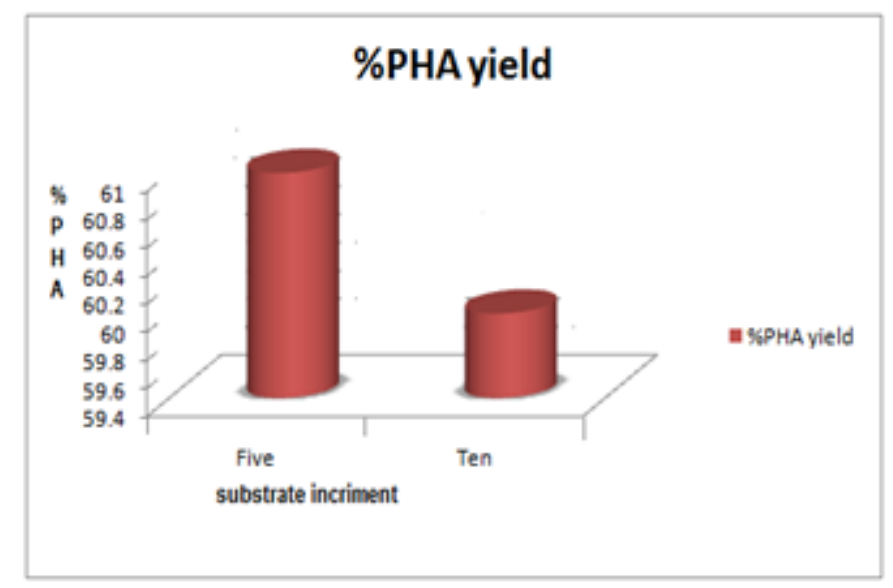

Percentage PHA yield by increase in substrate with combination of sugarcane bagasse With P.aeruginosa

Carbon source plays a very important role in the production of PHA. Increase in the concentration of carbon source by five times produced $61 \%$ of PHA and the production was reduced to $60 \%$ when the concentration was increased ten times. This is due to the attainment of saturation level, more concentration of carbon source lead to the death of micro organism.

Pseudomonas aeruginosa accumulated the maximum yield of 60\% PHA when sugarcane bagasse was used as carbon source. This result is very similar to the report of Ogunjobi, A.A.et al, (2011) who reported the yield of $55.68 \%$ by Pseudomonas putrefaciens with sugarcane baggasse. This result is also very similar to the report of Suzuki et al., (1986) who reported maximum yield of $66 \%$ w/w dry weight by a Pseudomonas sp. when methanol was used as sole carbon substrate for PHA production. The use of methanol as a carbon source is significant because it is such a cheap carbon source and could reduce production cost. However, sugarcane bagasse allowed the highest biomass density in this bacterial strain and the PHA yield obtained was equally high. This observation further supports the reports of Santhanam and Screenivasan, (2010) that a higher concentration of PHA polymer obtained with commercial sugar suggests the possibility of using cane molasses or other cheaper sucrose substrates in future.

\section{Conclusion}

PHA content and its composition are influenced mainly by the strain of microorganism, the type of substrate employed and its concentration, and environmental growth conditions. The production of biodegradable polyesters on a large scale is limited because of the relative expensive substrate required, low polymer production per unit of substrate utilized, and the cost of maintaining pure cultures. Higher production costs, especially raw material costs, make it difficult for PHA biodegradable plastics to compete with conventional petroleum-base plastics in the commercial marketplace. Sugarcane bagasse, a readily available bio-waste material was found to be the most suitable carbon substrate on selected bacterial species used in this study, as it enhanced the highest polymer yields. Hence, the production of these environmentally-friendly materials can compete with synthetic plastics on a large-scale if the cost of fermenting substrate, which contributes significantly to the overall production cost, has been reduced considerably. 


\section{References}

1. Caesar Preeti and Tiwari Archana(2010),in "Integration of natural and Biological sources for the Production of Biopolymer: Actual and Potential Utilization of various Wastes", Caesar Preeti et al. / Journal of Pharmacy Research 2011,4(1),53-55

2. Ching-Yee Loo and Kumar Sudesh(2007), in "Polyhydroxyalkanoates: Bio-based microbial plastics and their properties", Malaysian Polymer Journal (MPJ), Vol 2, No. 2, p 31-57, 2007.

3. M.C. Santimano, Nimali N. Prabhu and S. Garg, in "PHA Production Using Low-Cost Agro-Industrial Wastes by Bacillus sp. Strain COL1/A6.

4. Sathesh Prabu, C. and Murugesan, A . G(2009), in "Effective Utilization and Management of Coir Industrial waste for the Production of poly- $\beta$ - hydroxybutyrate (PHB) using the Bacterium Azotobacter Beijerinickii

5. Catalina VoaideS, Diana Groposila, Matilda Ciuca, Irina Lupescu, Aneta Pop, Calina Petruta Cornea(2010),in "PHAs accumulation in Pseudomonas putida P5 (wild type and mutants) in lipid containing media", Romanian Biotechnological Letters, Vol. 15, No. 4, 2010.

6. S.Ramalingam,M.Vikaram,M P Vignesh babu and M Sivasankari(2011), in "Flux Balance analysis for maximising polyhydroxy alkanoate production Pseudomonas putida”, Indian Journal of Biotechnology Vol 10, Jan 2011,pp 70-74.

7. Ogunjobi, A. A., Ogundele, A.O., and Fagade, O.E(2011), in "Production Of Polyhydroxyalkanoates By Pseudomonas Putrefaciens From Cheap And Renewable Carbon Substrates", EJEAFChe, 10(8), 2011 [2806-2815].

8. Hang Zang, Vincent Obias, Ken Goyer and Douglas Dennis(1994), in "Production of polyhydroxyalkanoates in sucroseutilising recombinant Escherichia coli and Klebsiella strains", Applied and Environmental Microbiology Vol 60, Apr 1994, p.1198-1205.

9. Rob AJ Verlinden, David J Hill, Melvin A Kenward, Craig D Williams, Zofia Piotrowska-Seget and Iza K Radecka(2011), in "Production of polyhydroxyalkanoates from waste frying oil by Cupriavidus necator", AMB Express 2011, 1:11.

10. Yoshikazu Kawata and Seiichi Aiba (2011). Patent application title: Method for producing PHAs using Halobacterium. Patent app no. 20110104767. 